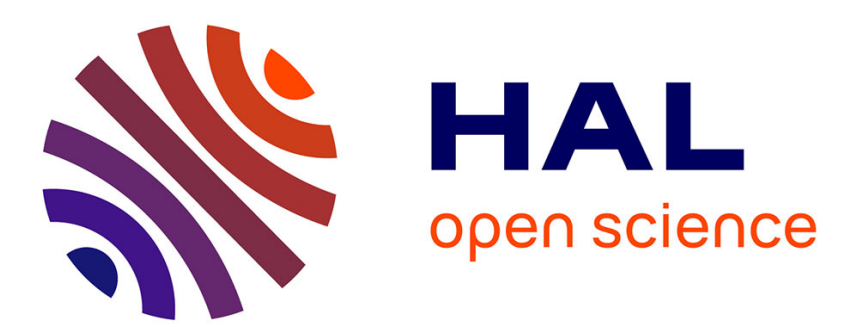

\title{
Global and bifurcation analysis of a structure with cyclic symmetry
}

Emmanuelle Sarrouy, Aurélien Grolet, Fabrice Thouverez

\section{To cite this version:}

Emmanuelle Sarrouy, Aurélien Grolet, Fabrice Thouverez. Global and bifurcation analysis of a structure with cyclic symmetry. International Journal of Non-Linear Mechanics, 2011, 46 (5), pp.727-737. 10.1016/j.ijnonlinmec.2011.02.005 . hal-00623630

\section{HAL Id: hal-00623630 \\ https://hal.science/hal-00623630}

Submitted on 14 Sep 2011

HAL is a multi-disciplinary open access archive for the deposit and dissemination of scientific research documents, whether they are published or not. The documents may come from teaching and research institutions in France or abroad, or from public or private research centers.
L'archive ouverte pluridisciplinaire HAL, est destinée au dépôt et à la diffusion de documents scientifiques de niveau recherche, publiés ou non, émanant des établissements d'enseignement et de recherche français ou étrangers, des laboratoires publics ou privés. 


\title{
Global and bifurcation analysis of a structure with cyclic symmetry
}

\author{
E. Sarrouy ${ }^{\mathrm{a}, *}$, A. Grolet $^{\mathrm{a}}$, F. Thouverez ${ }^{\mathrm{a}}$ \\ ${ }^{a}$ Ecole Centrale de Lyon, Laboratoire LTDS, Bat. E6, 36 avenue Guy de Collongue, 69134 Ecully Cedex, France
}

\begin{abstract}
This article combines the application of a global analysis approach and the more classical continuation, bifurcation and stability analysis approach of a cyclic symmetric system. A solid disc with four blades, linearly coupled, but with an intrinsic non-linear cubic stiffness is at stake. Dynamic equations are turned into a set of non-linear algebraic equations using the Harmonic Balance Method. Then periodic solutions are sought using a recursive application of a global analysis method for various pulsation values. This exhibits disconnected branches in both the free undamped case (non-linear normal modes, NNMs) and in a forced case which shows the link between NNMs and forced response. For each case, a full bifurcation diagram is provided and commented using tools devoted to continuation, bifurcation and stability analysis.
\end{abstract}

Keywords: Global analysis, Non-linear, Homotopy, Rotordynamics, Cyclic symmetry

\section{Introduction}

In this paper, both free and forced vibrations of a non-linear cyclic symmetric structure are studied. The structure is composed of $n_{b}$ identical substructures which undergo large strains. This system is typical when one studies bladed disks [2, 27]. After modeling the system, a set of coupled non-linear differential equations in which non-linearity appears by cubic terms is obtained.

In the linear case, the study of the linear normal modes (LNMs) reveals a majority of double eigenfrequencies, corresponding to distinct eigen-

\footnotetext{
*Corresponding author. Tel: +33 681393569/Fax:+33 144246468

Email addresses: emmanuelle.sarrouy@gmail.com (E.

Sarrouy), aurelien.grolet@ecl2009.ec-lyon.fr (A.

Grolet), fabrice.thouverez@ec-lyon.fr (F. Thouverez)
}

forms [22]. These eigenforms are associated with nodal diameter vibration modes. As these LNMs arise from an eigenvalue problem, there are always as many LNMs as degrees of freedom (dofs). Moreover, in the free or forced case, only one solution exists for a given frequency.

In the case of non-linear systems with cyclic symmetry, it has been shown that the number of nonlinear normal modes (NNMs, [7]) can exceed the number of dofs, the extra NNMs being generated through bifurcations or internal resonances [7, 28]. Localized non-linear modes are an example of this property; they correspond to a free motion in which only a few substructures vibrate with non negligible amplitude and they have no counterpart in the linear theory $[2,29]$. In the forced case, these additional NNMs give rise to a number of additional 
resonances leading to multiple solutions as in [29] where Vakakis showed a very complicated structure of resonance for a non-linear system with cyclic symmetry by using the multiple scales method. He showed that for a given frequency many solutions can coexist, some of them being stable.

Not only can multiple solutions coexist but also can they be disconnected from each other. In this latter case, classical methods based on continuation and bifurcation analysis fail at finding the disconnected branches of solutions. If one wants to dimension a structure properly - by considering all the possible solutions - one then needs to adopt a global analysis (GA) approach. Several solutions are mentioned in the literature (see [23] for an overview) with a common drawback even for small systems which is the computation cost. The GA method proposed in this paper takes advantage of the cubic form of the non-linearity combined with a reformulation of equations through the harmonic balance method (HBM); the resulting systems in the free undamped case for searching NNMs as well as in a forced case can then be solved (globally) in a reasonable amount of time.

Section 2 describes the system and its dynamical and HBM equations. The global analysis principle is then explained in section 3.1; this is followed by recalls on continuation methods, bifurcation and stability analysis in section 3.2. Finally these methods are applied to the undamped free system in order to find NNMs in section 4 and to a forced case which exhibits a very rich response in section 5 .

\section{Studied system}

\subsection{General description and dynamical equations}

The studied structure has a cyclic symmetry property and can therefore be broken up in $n_{b}$ identical sectors (Fig. 1). Each sector is modeled by a thin rectangular plate clamped to the rigid disk which itself is fixed (Fig. 2). Consecutive plates are coupled by a linear stiffness while nonlinearity is introduced by taking into account their large deflection. Plane stress assumption as well as the Love-Kirchhoff hypothesis (cross-sections exhibit solid body motion and remain perpendicular to the deformed surface of the middle sheet) are made. Then, plates displacements are entirely parameterized by their middle sheet transverse displacement $w_{j}, 1 \leq j \leq n_{b}$. Moreover the material is assumed to follow a standard bi-dimensional Hooke's law leading to the following expression for the strain energy of plate $j$ :

$$
\begin{aligned}
U_{j} & =\frac{1}{2} \frac{E h}{\left(1-\nu^{2}\right)} \int_{x=0}^{L_{x}} \int_{y=-L_{y} / 2}^{L_{y} / 2}\left[\left(\frac{1}{2}\left(\frac{\partial w_{j}}{\partial x}\right)^{2}\right)^{2}\right. \\
& +\left(\frac{1}{2}\left(\frac{\partial w_{j}}{\partial y}\right)^{2}\right)^{2}+2 \nu\left(\frac{1}{2}\left(\frac{\partial w_{j}}{\partial x}\right)^{2} \frac{1}{2}\left(\frac{\partial w_{j}}{\partial y}\right)^{2}\right) \\
& \left.+\frac{1-\nu}{2}\left(\frac{\partial w_{j}}{\partial x} \frac{\partial w_{j}}{\partial y}\right)^{2}\right] d x d y \\
& +\frac{1}{2} \frac{E h^{3}}{12\left(1-\nu^{2}\right)} \int_{x=0}^{L_{x}} \int_{y=-L_{y} / 2}^{L_{y} / 2}\left[\left(\frac{\partial^{2} w_{j}}{\partial x^{2}}\right)^{2}\right. \\
& +\left(\frac{\partial^{2} w_{j}}{\partial y^{2}}\right)^{2}+2 \nu\left(\frac{\partial^{2} w_{j}}{\partial x^{2}} \frac{\partial^{2} w_{j}}{\partial y^{2}}\right) \\
& \left.+\frac{1-\nu}{2}\left(2 \frac{\partial^{2} w_{j}}{\partial x \partial y}\right)^{2}\right] d x d y
\end{aligned}
$$

where $E$ is the Young's modulus and $\nu$ is the Poisson's ratio. 
The energy $V^{j}$ of the linear stiffness localized at $\left(x_{r}, y_{r}\right)=\left(L_{x} / 4,0\right)$ between plates $j$ and $j+1$ is given by:

$$
V_{j}=\frac{1}{2} k\left(w_{j}\left(x_{r}, y_{r}\right)-w_{j+1}\left(x_{r}, y_{r}\right)\right)^{2}
$$

for $1 \leq j \leq n_{b}$ with convention $j+1=1$ if $j=n_{b}$.

By neglecting rotary inertia the kinetic energy $T_{j}$ of plate $j$ is given by :

$$
T_{j}=\frac{1}{2} \rho h \int_{x=0}^{L_{x}} \int_{y=-L_{y} / 2}^{L_{y} / 2} \dot{w}_{j}^{2} d x d y
$$

In this paper, only an harmonic force, orthogonal to the plate, localized in $\left(x_{f}, y_{f}\right)=\left(L_{x}, 0\right)$ is considered. The work $W_{j}$ due to such an excitation on plate $j$ is given by:

$$
W_{j}=w_{j}\left(L_{x}, 0\right) \mathbf{F}_{\mathrm{e} j}
$$

The total energies $U, T, V$ and $W$ are then given by the sum over the number of plates $n_{b}$ of the different local energies $U_{j}, T_{j}, V_{j}$ and $W_{j}$. Equations of motions are finally derived by using Lagrange's equations along with a Rayleigh-Ritz approximation $[16]$.

[Figure 1 about here.]

[Figure 2 about here.]

The remainder of this article will be devoted to studying the case of a system composed of $n_{b}=4$ identical sectors, whose displacements are interpolated by a single Ritz function approximating the first bending mode thus yielding a non-linear problem with $n=4$ dofs that will serve as an example for the present work. The Ritz function is $\Phi(x, y)=\left(\frac{x}{L_{x}}\right)^{2}$ (consistent with the clamping at $x=0$ ) and leads to the following interpolation for transverse displacement of the $j$-th blade:

$$
w_{j}(x, y)=q_{j} \Phi(x, y) \text { for } 1 \leq j \leq n_{b}
$$

By applying Lagrange's equations, and by adding a damping term, the following motion equations are obtained:

$$
[\mathbf{M}] \ddot{\mathbf{X}}+[\mathbf{C}] \dot{\mathbf{X}}+[\mathbf{K}] \mathbf{X}+\beta \mathbf{X}^{3}=\mathbf{F}_{\mathrm{e}}(t)
$$

with the notations $\mathbf{X}=\left(q_{j}\right)_{1 \leq j \leq n}$, and $\mathbf{X}^{3}=$ $\left(q_{j}^{3}\right)_{1 \leq j \leq n}$. The vector $\mathbf{F}_{\mathrm{e}}=\mathbf{F}_{\mathrm{e} \mathbf{0}} \cos (\omega t)$ stands for the external forces amplitude, $[\mathbf{M}]=[\mathbf{I}]$ is the mass matrix, $[\mathbf{C}]=\delta[\mathbf{I}]$ is the damping matrix, $[\mathbf{K}]$ is the stiffness matrix given by:

$$
[\mathbf{K}]=\left[\begin{array}{cccc}
\alpha+2 c & -c & 0 & -c \\
-c & \alpha+2 c & -c & 0 \\
0 & -c & \alpha+2 c & -c \\
-c & 0 & -c & \alpha+2 c
\end{array}\right]
$$

and $\beta$ is the non-linear stiffness coefficient. The definitions of $\alpha, \beta, c$, and $\delta$ can be found in A along with their numerical values.

\subsection{Harmonic balance method}

The harmonic balance method (HBM) is widelyused for the study of non-linear systems. Numerous applications can be found in the literature, showing its ability to treat strongly non-linear systems like friction between blades and casing $[11,10]$ or geometric non-linearities [14, 21]. One major advantage of the method is that it requires no assumption about the non-linearities magnitudes and uses the same procedure for strongly and weakly non-linear models. 
The HBM consists in a decomposition of the solution $\mathbf{X}$ in a truncated Fourier series:

$$
\mathbf{X}(t)=\mathbf{A}_{0}+\sum_{k=1}^{N_{h}} \mathbf{A}_{k} \cos (k \omega t)+\mathbf{B}_{k} \sin (k \omega t)
$$

Injecting this development (8) in equation (6), and by projecting equations on the $\left[1,(\cos (k \omega t), \sin (k \omega t))_{1 \leq k \leq N_{h}}\right] \quad$ basis using the following scalar product

$$
\langle f, g\rangle=\int_{0}^{\frac{2 \pi}{\omega}} f(t) g(t) d t
$$

one gets a system of $\tilde{n}=n \times\left(2 N_{h}+1\right)$ non-linear algebraic equations with $n \times\left(2 N_{h}+1\right)+1$ unknowns $\mathbf{A}_{k}, \mathbf{B}_{k}$ and $\omega$.

The number of harmonics retained $N_{h}$ is a very important parameter. Generally, the higher $N_{h}$ is, the better the solution. However, in the case where the number of harmonics selected is high, the solution procedure can quickly become difficult and time consuming. Fortunately, in most cases the series converges fast enough and leads to systems with reasonable dimensions. In this article only the first harmonic is going to be retained $\left(N_{h}=1\right)$ and the constant term $\mathbf{A}_{0}$ is dropped due to the symmetry of the system. Depending on whether the system is free or forced, different formulations can be obtained.

\section{Forced case}

The equation to be solved in the forced case is given by (6). The solution $\mathbf{X}$ is sought of the following form:

$$
\mathbf{X}(t)=\mathbf{A} \cos (\omega t)+\mathbf{B} \sin (\omega t)
$$

which leads to the following set of $2 n$ algebraic equations:

$$
\left[\mathbf{H}_{1}\right] \tilde{\mathbf{X}}+\mathbf{H}_{\mathrm{nl}}(\tilde{\mathbf{X}})=\mathbf{H}_{\mathrm{e}}
$$

where $\tilde{\mathbf{X}}=\left\{\begin{array}{l}\mathbf{A} \\ \mathbf{B}\end{array}\right\}$ is the unknown in the frequency domain; it follows that $\tilde{n}=4 \times 2=8$. $\left[\mathbf{H}_{4}\right]$ corresponds to the linear part of the HBM:

$$
\left[\mathbf{H}_{1}\right]=\left[\begin{array}{cc}
{[\mathbf{K}]-\omega^{2}[\mathbf{M}]} & \omega[\mathbf{C}] \\
-\omega[\mathbf{C}] & {[\mathbf{K}]-\omega^{2}[\mathbf{M}]}
\end{array}\right]
$$

$\mathbf{H}_{\mathrm{e}}$ corresponds to the constant part related to the excitation

$$
\mathbf{H}_{\mathrm{e}}=\left\{\begin{array}{c}
\mathbf{F}_{\mathrm{e} \mathbf{0}} \\
\mathbf{0}
\end{array}\right\}
$$

and $\mathbf{H}_{\mathrm{nl}}$ corresponds to the non-linear part

$$
\begin{aligned}
& \mathbf{H}_{\mathrm{nl} i}(\tilde{\mathbf{X}})=\frac{3}{4} \beta\left(\mathbf{A}_{i}^{3}+\mathbf{A}_{i} \mathbf{B}_{i}^{2}\right) \\
& \mathbf{H}_{\mathrm{nl} n+i}(\tilde{\mathbf{X}})=\frac{3}{4} \beta\left(\mathbf{B}_{i}^{3}+\mathbf{B}_{i} \mathbf{A}_{i}^{2}\right)
\end{aligned} \quad \text { for } 1 \leq i \leq n
$$

Notice that in this case the solution frequency $\omega$ is taken equal to the one of the excitation force.

\section{Free Case}

The equation to be solved in the free undamped case is given by:

$$
[\mathbf{M}] \ddot{\mathbf{X}}+[\mathbf{K}] \mathbf{X}+\beta \mathbf{X}^{3}=\mathbf{0}
$$

and the solution $\mathbf{X}$ is sought of the following form:

$$
\mathbf{X}(t)=\mathbf{A} \cos (\omega t)
$$

The fact that only the cosine terms are retained corresponds to a phase condition in which all initial velocities are set to zero. With this approximation for the solution $\mathbf{X}, \tilde{n}=4$ and the following set of algebraic equations is obtained:

$$
\left([\mathbf{K}]-\omega^{2}[\mathbf{M}]\right) \mathbf{A}+\frac{3}{4} \beta \mathbf{A}^{3}=\mathbf{0}
$$

As there is no excitation force, the free solution frequency $\omega$ is also an unknown. 


\section{Theory}

This section is devoted to theory on both global analysis (GA) aspects and continuation coupled to stability and bifurcation analysis.

\subsection{Global analysis}

When aiming at finding all the solutions of a nonlinear system, looking at the whole state space seems the right way to proceed. This is what most global analysis methods such as cell-mapping [5], cell exclusion algorithm [32] or algorithms based on interval analysis [4] do. The main drawback of all these methods is their huge cost with regards to their computational resource consumption (see [23] for a comparison). In order to bypass this problem, one can take advantage of the algebraic form of our problem formulated in the frequency space and use a dedicated method to solve it globally relying on homotopy $[25,23,24,15]$. Indeed, the nonlinearity introduced being cubic, equations (11) or (17) can be considered as polynomials in $\tilde{\mathbf{X}}$ variable. We will now denote $\mathbf{P}$ the corresponding polynomial.

The main idea of this method is to embed the polynomial $\mathbf{P}$ to solve in a space whose parameters are the polynomial coefficients. Then, starting from a polynomial $\mathbf{Q}$ whose zeros (in $\mathbb{C}^{\tilde{n}}$ ) are easy to compute, a continuation scheme is applied to slide smoothly from these zeros to the ones of interest by substituting slowly $\mathbf{P}$ to $\mathbf{Q}$ which is, namely, applying a homotopy method. The system solved is

$$
\mathbf{R}(\tilde{\mathbf{X}}, \lambda)=\lambda \mathbf{Q}(\tilde{\mathbf{X}})+(1-\lambda) \mathbf{P}(\tilde{\mathbf{X}}), \lambda \in[0,1]
$$

with $\lambda$ varying from 1 to 0 .
In order to be able to guarantee that all $\mathbf{P}$ zeros will be reached, one has to respect a few rules: first of all, as each non-singular zero of $\mathbf{P}$ must be linked to a non-singular zero of $\mathbf{Q}$, the initial polynomial must have at least as many zeros as $\mathbf{P}$. Then, the paths linking $\mathbf{Q}$ zeros to $\mathbf{P}$ zeros must be smooth (undergo no bifurcation when $\lambda$ slides from 1 to 0 ) which implies that both polynomials must share structural properties [25, Chap. 8]. A basic polynomial which has easy to find zeros and respects these rules is

$$
\mathbf{Q}(\tilde{\mathbf{X}})=\gamma\left\{\begin{array}{c}
\tilde{\mathbf{X}}_{1}^{d_{1}}-1 \\
\vdots \\
\tilde{\mathbf{X}}_{\tilde{n}}^{d_{\tilde{n}}}-1
\end{array}\right\}, \gamma \in \mathbb{C}, d_{i}=\operatorname{deg}\left(\mathbf{P}_{i}\right)
$$

Such a polynomial has $\mathcal{N}=\prod_{i=1}^{\tilde{n}} d_{i}$ zeros, that is it has the maximum number of zeros a polynomial with such total degrees (maximum degree $d_{i}$ among its monomials for each component $\mathbf{P}_{i}$ ) can have. A homotopy process with this kind of initial polynomial is called a total degree homotopy. The total degree homotopy theorem demonstrated in $[25$, chap. 8, p. 123] ensures that the $\mathcal{N}$ zeros of $\mathbf{Q}$ will lead to all the non-singular zeros of $\mathbf{P}$. In our frame, only zeros with real components will be considered.

In many cases, $\mathbf{P}$ has only a few complex zeros. Starting with a lot more means a waste of time following paths that will mostly diverge towards infinity (as a homogeneization technique is used, they will remain numerically bounded, see $[25,31])$. That is why, in most cases it is interesting to work on the starting polynomial to reduce the number of starting zeros while still respecting the rules enunciated previously. Most current work 
aims at exhibiting such systems and automatizing their construction $([17,18,30,25,6,13])$. As will be shown later in this paper, such work is useless in the studied case. Using polynomial $\mathbf{Q}$ defined in (19), $3^{8}=6561$ starting zeros will be generated in the forced case (HBM equation (11)) and only $3^{4}=81$ paths will be followed in the free one (HBM equation (17)).

\subsection{Continuation, bifurcation and stability}

In this section, the classical tools to perform continuation and bifurcation analysis of fixed points (solutions in frequency space are constant) as well as the stability analysis of periodic solutions relying on Floquet coefficients evaluation are briefly recalled.

\subsubsection{Continuation algorithm}

A general system of equations used by a continuation algorithm is

$$
\mathbf{G}(\mathbf{Y}, \mu)=0
$$

where $\mathbf{G}$ is a non-linear function, $\mathbf{Y}$ is the vector of unknowns and $\mu$ is the continuation parameter. In the present case $\mathbf{Y}=\tilde{\mathbf{X}}$, the HBM unknowns and $\mu=\omega$, the free or forced frequency. The continuation procedure builds the path solution of (20) for some $\mu$ range by iterating two steps. First, a point is predicted using one or more of the previous points of the path, yielding an estimation of the next point. This predicted solution is then corrected iteratively until convergence is achieved.

In this study an arc-length continuation is used: $\mathbf{G}$ is obtained by augmenting the HBM set of equations (11) or (17) with an equation constraining the next point to rely at a given distance from the previous one. The prediction step is performed using a tangent predictor and the correction one uses a Newton-Raphson algorithm. More details about continuation techniques can be found in [1].

\subsubsection{Bifurcation detection}

In this paragraph, we will focus on turning points and branching points which are the main bifurcations encountered in this study. The detection of these bifurcation points is done by monitoring the determinant of the Jacobian matrix $\left[\mathbf{J}_{\mathbf{Y}}\right]=\frac{\partial \mathbf{G}}{\partial \mathbf{Y}}$ of (20): this determinant is null for such bifurcation points [8]. A Newton like algorithm, described in [19], is used to determine accurately the solutions $\left(\mathbf{Y}_{b}, \omega_{b}\right)$ such that

$$
\begin{aligned}
& \mathbf{G}\left(\mathbf{Y}_{b}, \omega_{b}\right)=0 \\
& \frac{\partial \mathbf{G}}{\partial \mathbf{Y}}\left(\mathbf{Y}_{b}, \omega_{b}\right) \mathbf{T}=0 \\
& \|\mathbf{T}\|=1
\end{aligned}
$$

where $\mathbf{T}$ is the tangent direction.

The type of bifurcation is then determined by estimating the rank of the Jacobian matrix $[\mathbf{J}]=$ $\left[\begin{array}{ll}\mathbf{J}_{\mathbf{Y}} & \mathbf{J}_{\omega}\end{array}\right]$ (where $\left.\left[\mathbf{J}_{\omega}\right]=\frac{\partial \mathbf{G}}{\partial \omega}\right)$ which is of size $\tilde{n} \times$ $(\tilde{n}+1)$. This rank is exactly $\tilde{n}$ for turning points and no further work has to be done since the arclength continuation scheme is able to handle such points. In the case of branching point bifurcations, this rank is at most $\tilde{n}-1$ and several linearly independent tangents $\mathbf{T}_{j}$ respecting $\left[\mathbf{J}_{\mathbf{Y}}\right] \mathbf{T}_{j}=0$ can be exhibited. Each of them corresponds to a prediction direction to explore. In this study, the prediction directions are computed using the eigenvectors $\Psi_{i}$ of $\left[\mathbf{J}_{\mathbf{Y}}\right]$ associated with the zero eigenvalue at the bifurcation point [20]. 


\subsubsection{Stability using Floquet theory}

The stability of solutions is determined by using Floquet's theory. In this study, just the primary results of this method will be recalled; a more detailed presentation is available in [19].

To do so, the first order formulation of the dynamic system (6) is considered:

$$
\dot{\mathbf{U}}=\mathbf{f}(\mathbf{U}, t)
$$

with $\mathbf{U}$ a vector combining $\mathbf{X}$ and $\dot{\mathbf{X}}$.

If $\mathbf{U}^{*}(t)$ is a periodic solution of (22) with period $T^{*}$ and $\mathbf{z}(t)$ is a small perturbation added to $\mathbf{U}^{*}$, the equation governing its evolution is derived from (22):

$$
\dot{\mathbf{z}}=\frac{\partial \mathbf{f}}{\partial \mathbf{U}}\left(\mathbf{U}^{*}, t\right) \mathbf{z}
$$

which is linear with periodic coefficients. Therefore, any solution $\mathbf{z}(t)$ corresponding to any initial perturbation is a linear combination of $\mathbf{m}^{(i)}(t)$, solutions of (23) at time $t$ having all its components but the i-th null at time $t=0: \mathbf{m}_{j}^{(i)}(0)=\delta_{i j}$, $1 \leq i, j \leq 2 n$. The growth of $\mathbf{z}$ is then directly related to the one of $\mathbf{m}^{(i)}$ which is in turn evaluated via the eigenvalues of the monodromy matrix

$$
[\mathbf{M o n}]=\left[\mathbf{m}^{(1)}\left(T^{*}\right), \ldots, \mathbf{m}^{(2 n)}\left(T^{*}\right)\right]
$$

This was demonstrated and stated properly in the Floquet's theorem (1883). Eigenvalues of the monodromy matrix are usually complex and are called Floquet coefficients. They give us information on the stability of the solution $\left(\mathbf{U}^{*}\right.$ is unstable as soon as one of them has a magnitude greater than 1) and on the type of bifurcation (through the way they cross the unit circle in complex plane) [19, 8].

Some other techniques $[12,9]$ exploit the HBM results directly to determine the cycle stability. The authors confronted two methods. First a computation using Floquet basic theory relying on time integrations; the cycle $\mathbf{U}^{*}$ is then rebuilt using the HBM coefficients. Second, the perturbation technique proposed by $\mathrm{D}$. Laxalde in his $\mathrm{PhD}$ work [9] was implemented. Each time, both methods gave the same results.

\section{Application to backbone curves}

The aim of this section is to build the NNMs of the undamped structure (17). The results will be displayed mainly in the Frequency Energy Plot (FEP) which is one of the most appropriate ways to depict the frequency-energy dependence of such modes [7, 3]. First, a brief recall of the linear study results is proposed. Then, a global analysis step is performed and exhibits many solutions which are grouped with respect to their energy, creating energy branches. Each solution branch is finally followed and analyzed using tools of section 3.2. One of the branches and its bifurcations is analyzed in a detailed way before the drawing of the full bifurcation diagram.

\subsection{Linear system}

The linear system has the following pair of eigenvector and eigenvalues (eigenvectors normalized with respect to mass matrix), i.e. linear normal modes (LNM) :

$$
\begin{aligned}
& \omega_{1}=93.63 \mathrm{rad} . \mathrm{s}^{-1}, \mathbf{L}_{1}=\{1,1,1,1\}^{t} / 2 \\
& \omega_{2}=95.20 \mathrm{rad} . \mathrm{s}^{-1}, \mathbf{L}_{\mathbf{2}}=\{1,0,-1,0\}^{t} / \sqrt{2} \\
& \omega_{2}=95.20 \mathrm{rad} . \mathrm{s}^{-1}, \mathbf{L}_{3}=\{0,-1,0,1\}^{t} / \sqrt{2} \\
& \omega_{4}=96.75 \mathrm{rad} . \mathrm{s}^{-1}, \mathbf{L}_{4}=\{-1,1,-1,1\}^{t} / 2
\end{aligned}
$$


Using classical denominations [26], one can state that

- $\mathbf{L}_{\mathbf{1}}$ is a "mode 0" were all the coordinates have the same amplitude. This is a non-degenerate mode with no nodal diameter.

- $\mathbf{L}_{2}$ and $\mathbf{L}_{3}$ are degenerate "cos- 1 " and "sin-1" modes with 1 nodal diameter. According to [26, p. 202], the corresponding NNMs should have the same backbone $(n=4 \times p \times 1)$.

- $\mathbf{L}_{4}$ is a "mode $\mathrm{n} / 2$ " that exists because the system has an even number of blades. All coordinates vibrate with the same amplitudes but opposite phases, generating 2 nodal diameters.

These notations will be useful for backbone analysis in section 4.3 .

\subsection{Global analysis}

A global analysis method as explained in 3.1 was applied to the free system (17) in order to build backbone curves. As the system is already in a polynomial form, no further work was done on equations. The total degree homotopy method was applied recursively for $\omega$ between 93.0 rad.s ${ }^{-1}$ and 100.0 rad. $\mathrm{s}^{-1}$ with a 0.1 rad. $\mathrm{s}^{-1}$ step. For each step $3^{4}=81$ paths are followed. This computation took $758 \mathrm{~s}$ (about $12 \mathrm{~min} 38 \mathrm{~s}$ using an Intel Core 2 Duo E8400 (3Go RAM)) that is about $11 \mathrm{~s}$ per step and led to a great amount of solutions as depicted in Fig.

3. Besides the great number of solutions, one can see that plots for each $\mathbf{A}_{i}$ look similar which is in accordance with the cyclic symmetry of the structure. Each solution returned by the global analysis algorithm was refined using a local Newton-Raphson method with a drastic $10^{-12}$ criterion on residual; no difference between raw GA results and refined ones appeared. We then ensured that each solution kept by the global analysis algorithm was different from the others from each step and counted them: there are up to 81 solutions for pulsations higher than 99.4 rad. $^{-1}$ ! This justifies the use of the basic total degree homotopy method (same number of zeros for $\mathbf{Q}$ and $\mathbf{P}$ ). This step also exhibited disconnected solutions. To have a better understanding of these numerous solutions, they were grouped with respect to their energy level. These energy levels are represented in a FEP, drawing branches that are named, as shown on Fig. 4. Each branch is named n.p $p_{1} \cdot p_{2}$. n denotes the number of nodal diameters of the main branch and a. $\mathrm{p}_{i}$ extension is added each time a bifurcated branch occurs. d1 and d2 refers to the disconnected branches and NS to the null solution. Symbols on this figure refer to the number of solutions that have the same energy level for a given pulsation $\omega$.

[Figure 3 about here.]

[Figure 4 about here.]

\subsection{Branches analysis using continuation}

Every solution corresponding to each energy branch has been continued and analyzed with regards to its stability and bifurcations using tools of section 3.2. As the previous global analysis step provided startpoints for each solution branch, the structure symmetry was not used to reduce the number of solutions to study; this would indeed have required a fine bifurcation analysis in conjunction with the symmetry consideration to determine 
the number of solutions appearing at each bifurcation point. As discussing each branch would be too long, one proposes to focus on branch 1' and its bifurcated branches. The same analysis was carried on for each branch and the results are summed up in subsection 4.4 .

For each energy branch 1', 1'.1 and 1'.1.1, one of the solutions matching this energy evolution is picked up as a representative of all the other solutions with the same energy evolution. Each time, a figure divided in four frames presents its HBM components evolution along with $\omega$ in frame (a); in frame (b) the evolution of its decomposition on LNM in percent is drawn: the solution components are projected on LNM of subsection 4.1 and these "modal coordinates" are brought back to percent by dividing by the sum of their absolute value and multiplying this by 100 . This will let us conclude on similar or non-similar property of the mode. Finally, Floquet coefficients are plotted in frames (c) and $(\mathrm{d})$ : frame $(\mathrm{c})$ shows their norm along $\omega$ while frame (d) represents them in the complex plane superimposed on the unit circle. In order to get accurate Floquet coefficients, solutions initially computed using only the first harmonic $\mathbf{A}_{1}$ were refined using $\mathbf{A}_{1}, \mathbf{A}_{3}$ and $\mathbf{A}_{5}$ components.

Branch 1' matches 4 different solutions with the same energy that contain both $\mathbf{L}_{\mathbf{2}}$ and $\mathbf{L}_{\mathbf{3}}$ contributions with equal weight (Fig. $5(\mathrm{a})$ ). These 4 solutions are identical with respect to a circular shift of tha $\mathbf{A}_{i}$ components. Fig. 5 (b) shows that it is a similar mode. Fig. 5 (c) and (d) show that this mode bifurcates and become unstable for $\omega_{3}=95.98 \mathrm{rad} . \mathrm{s}^{-1}$ generating one new energy branch, namely branch 1'.1. In fact, each of the 4 solutions underlying energy branch 1' bifurcates and gives birth to two half branches that match the branch 1'.1 energy level.

[Figure 5 about here.]

Branch 1'.1 matches 8 solutions that come from branch 1' bifurcation. These solutions include a LNM $\mathbf{L}_{1}$ contribution (Fig. 6 (b)) that becomes greater as $\omega$ increases. This indicates that this is not a similar mode. This branch is first unstable and becomes stable for $\omega_{4}=96.75$ rad.s ${ }^{-1}$, which is the frequency at which branch 2 arises. It then gives birth to a new branch (branch 1'.1.1) that "takes advantage" on the existence of this new energy level (of branch 2) as explained in the next paragraph. In the state space, each of the 8 half branches matching branch 1'.1 energy branch bifurcates once and gives birth to 2 new half branches. Solutions underlying this energy branch 1'.1 will bifurcate once again for $\omega_{8}=99.36 \mathrm{rad} . \mathrm{s}^{-1}$, that is for the same pulsation at which branches d1 and d2 arise.

[Figure 6 about here.]

Branch 1'.1.1 matches 16 solutions bifurcated from branch 1'.1 solutions. These solutions involve a new $\mathrm{LNM} \mathrm{L}_{\mathbf{4}}$ contribution that increases as the $\mathbf{L}_{1}$ contribution introduced in branch 1'.1 decreases. Fig. 7 (b) let us state this is a non-similar mode. This branch is always unstable.

\section{[Figure 7 about here.]}

Fig. 8 recapitulates branch 1' bifurcations by showing the four initial solutions that match this energy branch and the subsequent bifurcated solution branches. It is obvious via this figure that each 
initial solution and its bifurcated solutions (each line of plots in the figure) is equal to the others with respect to a circular shift of its components.

[Figure 8 about here.]

\subsection{Conclusion}

Table 1 and Fig. 9 summarize the results of the previous analysis carried out on all other branches. Fig. 9 exhibits branches similar to Fig. 4 but this time, points are linked continuously thanks to the continuation step. Moreover, this figure provides information on stability and not on the number of solutions sharing the same energy branch anymore.

This simple structure exhibits in fact a very rich modal behavior: a great number of solutions branches can be captured using continuation and bifurcation analysis. Due to cyclic symmetry, they draw a less important but still great number of energy branches. In addition to these numerous branches, the use of a GA method lets us intercept two disconnected branches $d 1$ and $d 2$ that would have been missed otherwise.

[Table 1 about here.]

[Figure 9 about here.]

\section{Application to a forced case}

The previous section exhibited numerous NNMs which will lead to complicated structures of resonance in forced cases. The aim of this section is to illustrate this complexity through an example.

The system is therefore excited with a force taking the branch 1' shape, thus vector $\mathbf{F}_{\mathrm{e} 0}$ of Eq. (13) is defined by $\mathbf{F}_{\mathrm{e} \mathbf{0}}=a_{f}\{1,1,-1,-1\}^{t}$. Simulations are carried out for $a_{f}=0.25 \mathrm{~N} . \mathrm{kg}^{-1}$.

First, the principal non-linear response has been computed by continuation. This solution, called Sol-A, corresponds to a motion with the 1' mode shape. The solution and its stability are represented in a FEP on Fig. 10.

By monitoring the determinant of the Jacobian matrix, 4 bifurcation points have been computed for Sol-A, they are also represented on Fig. 10 labeled from A1 to A4. By studying the rank of the Jacobian matrix, one can tell that points $\mathrm{A} 1$ and $\mathrm{A} 3$ are branching points $(\operatorname{rank}([\mathbf{J}])=7)$ and points $\mathrm{A} 2$ and A4 are not only turning points but also branching points $(\operatorname{rank}([\mathbf{J}])=6)$. Stable parts of the energy branch are denoted by a thick (blue) line on Fig. 10 while unstable ones are represented by a thin (red) one. Underlying NNMs are plotted using dashed dot (black) lines. Squared points denote branching points while circled ones indicate Hopf bifurcations. The same representation code is used for the following figures (Fig. 11 to Fig. 13). One can then see on Fig. 10 that sol-A is unstable even for relatively low energy levels.

[Figure 10 about here.]

Starting from A2 (or A4), the two bifurcated branches of solution have been computed (Sol-L2 and Sol-L3). The representation of these solutions in the amplitude frequency diagram is not very convenient because they superimpose themselves with a part of Sol-A, the only difference being that curves are not covered in the same way. To overcome this issue, we chose to represent these solutions in a FEP in Fig. 11. In this FEP, Sol-L2 and Sol-L3 
are superimposed, but one can see that these solutions possess stable parts and are positioned around backbone curves of modes with 1 nodal diameter (branch 1), therefore one can conclude that these two solutions correspond to resonances around nonlinear modes with shape L2 and L3.

[Figure 11 about here.]

Starting from A1 (or A3), a bifurcated branch has been computed (Sol-B), and its representation in the FEP is given on Fig. 12. Sol-B is positioned around the backbone curve branch 1'.1. A stability analysis reveals that Sol-B possesses stable parts and also unstable parts generated through Hopf bifurcation. Several branching points have been detected for Sol-B, namely B1, B2 and B3 (Fig. 12).

B2 (or B3) leads to an unstable bifurcated response, Sol-C, positioned around the backbone curve of branch 1'.1.1 (Fig. 13), and B1 leads to an unstable bifurcated response, Sol-D, which seems to be positioned close to the backbone curve of branch 1' (Fig. 13).

[Figure 12 about here.]

Finally, global analysis reveals another kind of solution, disconnected from the other. They are represented by closed curves in the FEP. This solution family, termed Sol-E, is positioned near the backbone branch 2.1 and is depicted on Fig. 13. Using formulation (11), 6561 paths are followed for each tested $\omega$. This takes approximately $1 \mathrm{~h} 15 \min$ per $\omega$ value using an Intel Core 2 Duo E8400 (3Go RAM) which seems reasonable with regard to the information it provides.

[Figure 13 about here.]
All the solutions are also depicted in a frequencyamplitude plot in Fig. 14 and Fig. 15. Two different figures were used for the sake of clarity. Only the amplitude of the first sector is considered.

[Figure 14 about here.]

[Figure 15 about here.]

In order to fully validate the stability analysis, direct numerical integrations have been performed. Initial conditions were provided by HBM solutions and simulations were carried out over 1000 periods. Results are plotted only for the five last periods. Fig. 16 (resp. Fig. 17) shows the temporal evolution of both numerical integration and HBM solution for a stable point of Sol-B (resp. Sol-E) at $\omega=99.53$ (resp. $\omega=98.84$ ). A good agreement can be observed for both simulations, thus confirming the validity of the proposed approach.

[Figure 16 about here.]

[Figure 17 about here.]

This example exhibits a very complicated structure of resonance which is closely related to the backbone curves previously presented. The FEP appears to be a convenient tool for identifying nonlinear modes involved in non-linear resonances.

Applied to this case, global analysis has two virtues: first of all, finding the disconnected solutions, that is guarantying that all the possible stable states will be considered when dimensioning the structure. The second one is not theoretical, but practical: if one can detect all the bifurcation points by carrying out a bifurcation analysis along the continuation process, it is easy to skip such points. For example, 
bifurcation points A2 and A4 being turning points with two additional tangent for each, the determinant of the Jacobian gets null but does not change sign. An automated script based on this criterion had therefore missed Sol-L2 and Sol-L3 branches and they were in fact found by the GA approach.

\section{Conclusion}

This paper applies both a classical continuation method and an original global analysis approach to a cyclic symmetric structure. These methods enable the drawing of a complete bifurcation diagram in a reasonable amount of time even in the case of numerous bifurcations and existence of disconnected solutions. Moreover it can intercept branches that would have been missed by the continuation and bifurcation analysis scheme. The forced case example also emphasizes the usefulness of NNMs to analyze a non-linear structure.

The ability of the proposed global analysis method to exhibit disconnected solutions being proved, further work now should focus on developments allowing its application to larger systems and to quasi-periodic solutions search.

\section{A. Definitions of parameters in the equation of motion}

This Appendix gives a symbolic and numeric expression for coefficients used in the equation of motion (6).

$$
\begin{aligned}
& m=\rho \int_{P} \Phi^{2} d P=\frac{\rho h L_{x} L_{y}}{5} \\
& c=\frac{k}{m} \Phi\left(x_{r}, y_{r}\right)^{2}=\frac{1}{256} \frac{k}{m} \\
& \mathbf{F}_{\mathrm{e} j}=\Phi\left(x_{f}, y_{f}\right) \frac{f^{j}(t)}{m}=\frac{f^{j}(t)}{m} \\
& \alpha=\frac{5}{3} \frac{E h^{2}}{\rho L_{x}^{4}\left(1-\nu^{2}\right)} \\
& \beta=\frac{8 E}{\rho L_{x}^{4}\left(1-\nu^{2}\right)}
\end{aligned}
$$

The damping coefficient $\delta$ is defined as:

$$
\delta=\frac{\sqrt{\alpha}}{200}
$$

Here are the numerical values used in this study: the geometric and physical parameters are affected by the following set of values:

$$
\begin{aligned}
& L_{x}=1.5 \mathrm{~m}, L_{y}=0.3 \mathrm{~m}, h=0.03 \mathrm{~m}, \\
& x_{r}=L_{x} / 4, y_{r}=0, x_{f}=L_{x}, y_{f}=0 \\
& E=210 \mathrm{GPa}, \nu=0.3, \rho=7800 \mathrm{~kg} \cdot \mathrm{m}^{-3}, \\
& k=8 \cdot 10^{5} \mathrm{~N} \cdot \mathrm{m}^{-1}
\end{aligned}
$$

which correspond to the following values for the different parameters in equation (6) :

$$
\begin{aligned}
& \alpha=8.7662 \cdot 10^{3} \mathrm{~s}^{-2}, \quad c=148.36 \mathrm{~s}^{-2}, \\
& \beta=4.6752 \cdot 10^{7} \mathrm{~m}^{-2} \cdot \mathrm{s}^{-2}
\end{aligned}
$$

\section{References}

[1] E. L. Allgower and K. Georg. Introduction to Numerical Continuation Methods. Springer-Verlag, 2003.

[2] Georgiades F., Peeters M., Kerschen G., and Golinval J.C. Modal analysis of a nonlinear periodic structure with cyclic symmetry. AIAA journal, 47:195-216, 2009.

[3] F. Georgiades, M. Peeters, Gaetan Kerschen, JeanClaude Golinval, and M. Ruzzene. Modal analysis of a nonlinear periodic structure with cyclic symmetry. AIAA, 47(4):1014-1025, 2009. 
[4] E. Hansen and G. W. Walster. Global Optimization Using Interval Analysis. M. Dekker, 2004.

[5] C.S. Hsu. Cell-to-Cell Mapping, a method of global analysis for non linear systems. Springer-Verlag, 1987.

[6] Birkett Huber and Jan Verschelde. Polyhedral end games for polynomial continuation. Numerical Algorithms, 18(1):91-108, 1998.

[7] G. Kerschen, M. Peeters, J.C. Golinval, and A.F. Vakakis. Nonlinear normal modes, part i: A useful framework for the structural dynamicist. Mechanical Systems and Signal Processing, 23(1):170 - 194, 2009. Special Issue: Non-linear Structural Dynamics.

[8] Y. A. Kuznetsov. Elements of Applied Bifurcation Theory, 3rd ed. Springer, 2004.

[9] D. Laxalde. Étude d'amortisseurs non-linaires appliqus aux roues aubages et aux systmes multitages. PhD thesis, École Centrale de Lyon, 2007. URL http://tel.archives-ouvertes.fr/tel-00344168/fr/.

[10] D. Laxalde and F. Thouverez. Complex nonlinear modal analysis for mechanical systems: application to turbomachinery bladings with friction interfaces. Journal of sound and vibration, 322:1009-1025, 2009.

[11] D. Laxalde, F. Thouverez, J. J. Sinou, and J. P. Lombard. Qualitative analysis of forced responce of blisks with friction ring dampers. European journal of mechanics and solids, 36:676-687, 2007.

[12] Arnaud Lazarus and Olivier Thomas. A harmonicbased method for computing the stability of periodic solutions of dynamical systems. Comptes Rendus Mcanique, 338(9):510 - 517, 2010. ISSN 1631-0721.

[13] T. Lee, T. Li, and C. Tsai. Hom4ps-2.0: a software package for solving polynomial systems by the polyhedral homotopy continuation method. Computing, 83 (2):109-133, 2008.

[14] R. Lewandowski. Computational formulation for periodic vibration of geometrically nonlinear structurespart 1:theoretical background. International Journal of Solids Structures, 34(15):1925-1947, 1997.

[15] T. Y. Li. Numerical solution of multivariate polynomial systems by homotopy continuation methods. Acta Numerica, 6:399-436, 1997.

[16] K.M. Liew and C.M Wang. pb2-rayleigh-ritz method for general plate analysis. Engineering structures, 15(1):
55-60, 1993.

[17] A. Morgan and A. Sommese. A homotopy for solving general polynomial systems that respects $\mathrm{m}$ homogenous structures. Applied Mathematics and Computation, 24:101-113, 1987.

[18] A. Morgan and A. Sommese. Coefficient-parameter polynomial continuation. Applied Mathematics and Computation, 29(2):123-160, 1989.

[19] A.H. Nayfey and B. Balanchandran. Applied nonlinear dynamics. Wiley-Interscience, 1995.

[20] P. Ribeiro and M. Petyt. Non-linear free vibration of isotropic plates with internal resonance. International Journal of Non-Linear Mechanics, 35:263-278, 2000.

[21] P. Ribiero and M. Petyt. Nonlinear vibration of plates by the hierarchical finite element and contination method. International journal of mechanical science, 41:437-459, 1999.

[22] S. Samaranayake. Subharmonic oscillations in harmonically excited mechanical systems with cyclic symmetry. Journal of Sound and Vibration, 206(1):39-60, 1997.

[23] E. Sarrouy and F. Thouverez. Global search of nonlinear systems periodic solutions: A rotordynamics application. Mechanical Systems and Signal Processing, In Press, Corrected Proof:-, 2010. doi: 10.1016/j. ymssp.2010.02.001.

[24] Emmanuelle Sarrouy. Analyse globale de systèmes mécaniques non-linéaires - Application à la dynamique des rotors. $\mathrm{PhD}$ thesis, École Centrale de Lyon, October 2008. URL http://tel.archives-ouvertes.fr/tel-00366857/fr/.

[25] A. J. Sommese and C. W. Wampler. The Numerical Solution of Systems of Polynomials Arising in Engineering and Science. World Scientific Publishing Co. Pte. Ltd., 2005.

[26] A.F. Vakakis. Dynamics of a nonlinear periodic structure with cyclic symmetry. Acta Mechanica, 95(1):197226, 1992.

[27] A.F. Vakakis. A multiple-scales analysis of nonlinear, localized modes in a cyclic periodic system. Journal of Applied Mechanics, 60:388-397, 1993.

[28] A.F. Vakakis. Nonlinear normal modes and their application in vibration theory: an overview. Mechanical systems and signal processing, 11(1):3-22, 1997. 
[29] A.F. Vakakis and A.F. King. A very complicated structure of resonances in a nonlinear system with cyclic symmetry: non linear forced localization. Nonlinear dynamics, 7:85-104, 1995.

[30] C. W. Wampler. Bézout number calculations for multihomogeneous polynomial systems. Applied Mathematics and Computation, 51(2-3):143-157, 1992.

[31] A. H. Wright. Finding all solutions to a system of polynomial equations. Mathematics of Computation, 44(169):125-133, 1985.

[32] Z-B. Xu, J-S. Zhang, and W. Wang. A cell exclusion algorithm for determining all the solutions of a nonlinear system of equations. Applied Mathematics and Computation, 80:181-208, 1996. 


\section{List of Figures}

Bladed wheel model . . . . . . . . 16

Rectangular plate diagram . . . . . . 17

GA applied to free system . . . . . . 18

GA for NNMs: energy branches . . . 19

Branch 1' analysis . . . . . . . . . . 20

Branch 1'.1 analysis . . . . . . . . . 21

Branch 1'.1.1 analysis . . . . . . . . . 22

Energy branch 1' sum up . . . . . 23

Backbones FEP with stability . . . . 24

10 Forced case: Sol-A . . . . . . . . . . 25

11 Forced case: Sol-A, Sol-L2 and Sol-L3 26

12 Forced case: Sol-A and Sol-B . . . . 27

13 Forced case: Sol-B, Sol-D and Sol-E 28

14 Forced case: sector 1 magnitude (1/2) 29

15 Forced case: sector 1 magnitude $(2 / 2) \quad 30$

16 Forced case: Comparison between HBM and numerical integration (1/2) 31

17 Forced case: Comparison between HBM and numerical integration (2/2) 32 


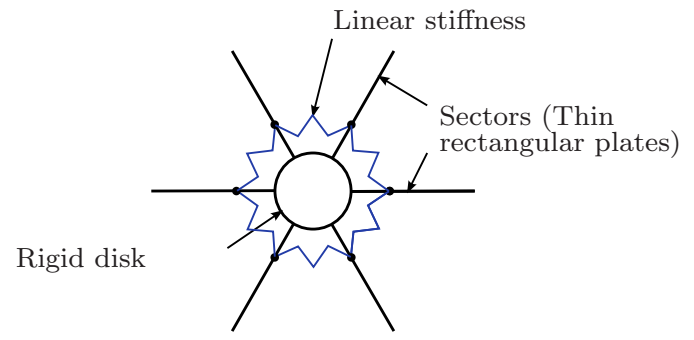

Figure 1: Bladed wheel model used for establishing equations 


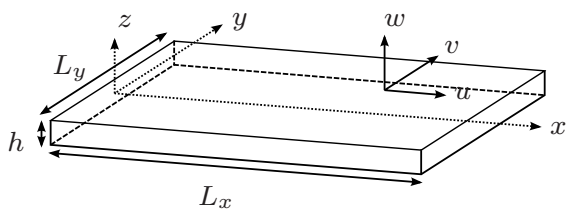

Figure 2: Diagram of a rectangular plate and corresponding coordinate system 

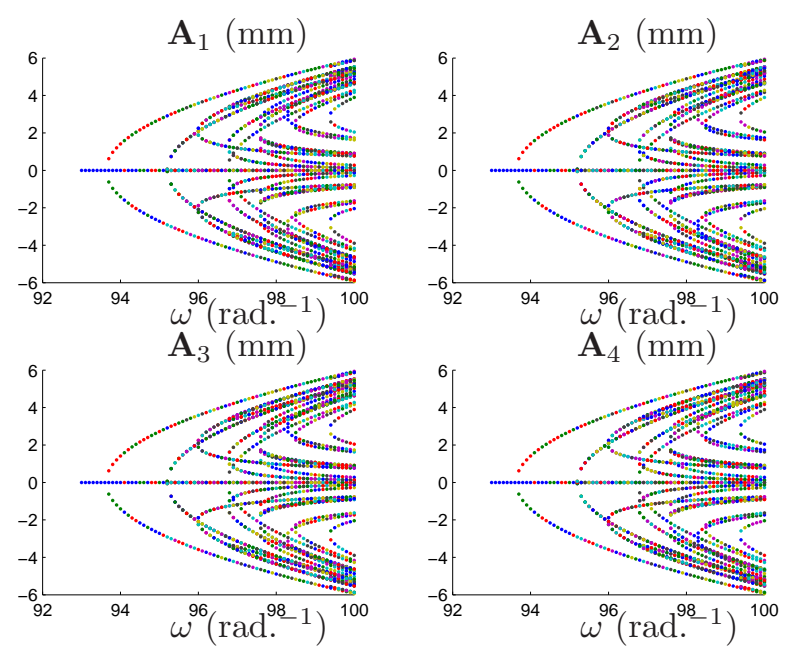

Figure 3: GA applied to free system: solutions components 


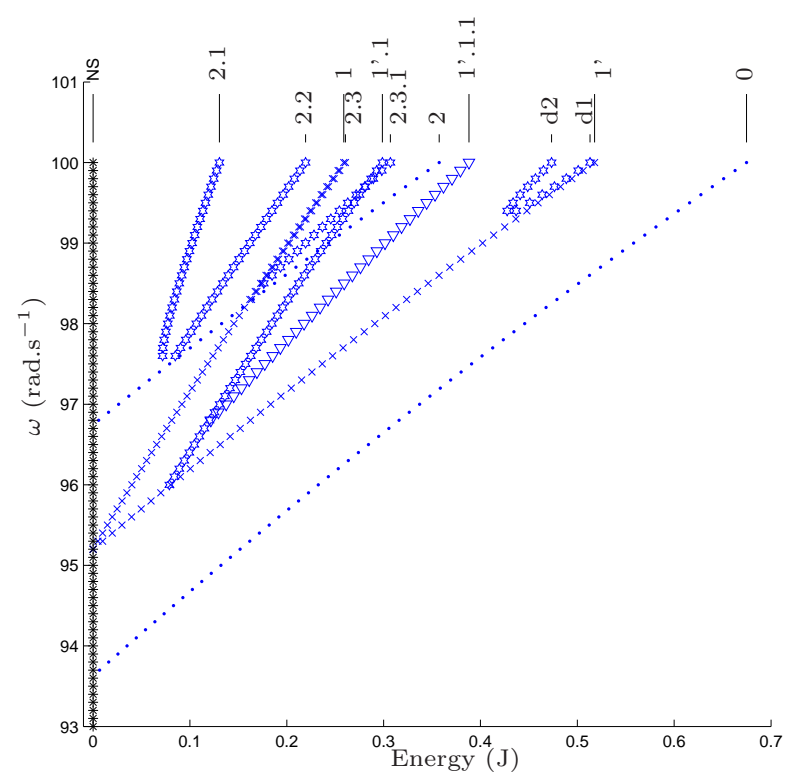

* 1 sol. . 2 sol. $\times 4$ sol. 8 sol. $\nabla 16$ sol.

Figure 4: GA for NNMs: energy branches 
(a) $\mathbf{A}_{i}(\mathrm{~mm})$

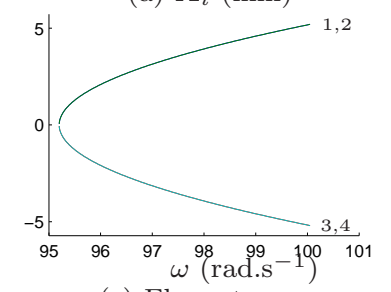

(c) Floquet: norm

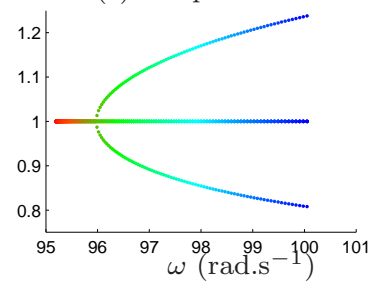

(b) Modal Decompo. (\%)

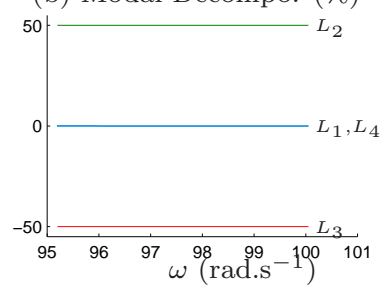

(d) Floquet: $\mathbb{C}$ plane

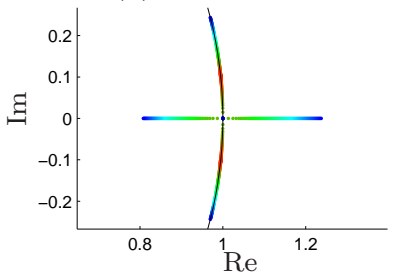

Figure 5: Branch 1' analysis 
(a) $\mathbf{A}_{i}(\mathrm{~mm})$

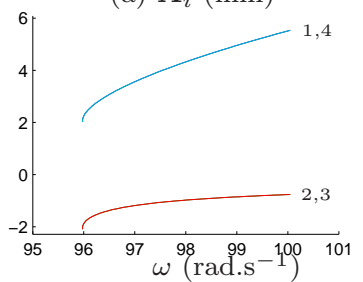

(c) Floquet: norm

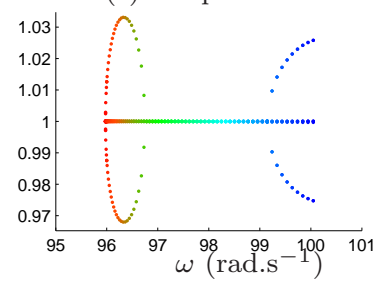

(b) Modal Decompo. (\%)

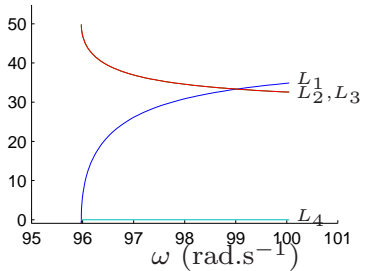

(d) Floquet: $\mathbb{C}$ plane

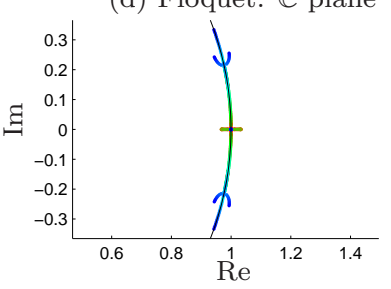

Figure 6: Branch 1'.1 analysis 
(a) $\mathbf{A}_{i}(\mathrm{~mm})$

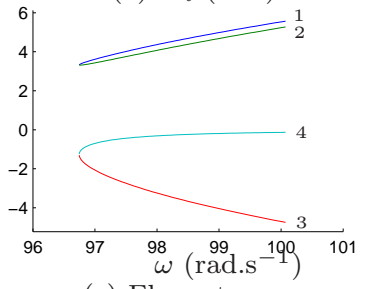

(c) Floquet: norm

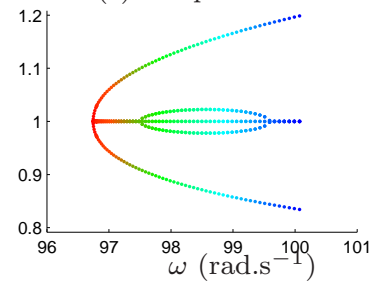

(b) Modal Decompo. (\%)

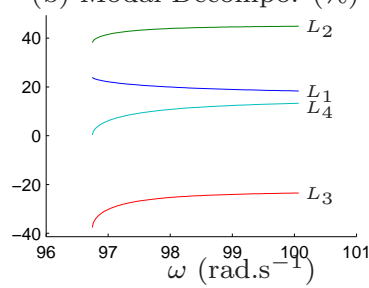

(d) Floquet: $\mathbb{C}$ plane

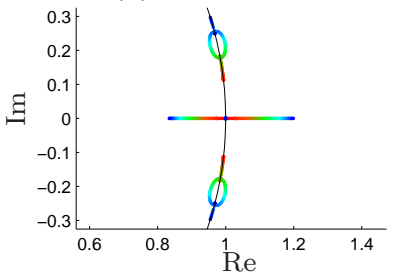

Figure 7: Branch 1'.1.1 analysis 

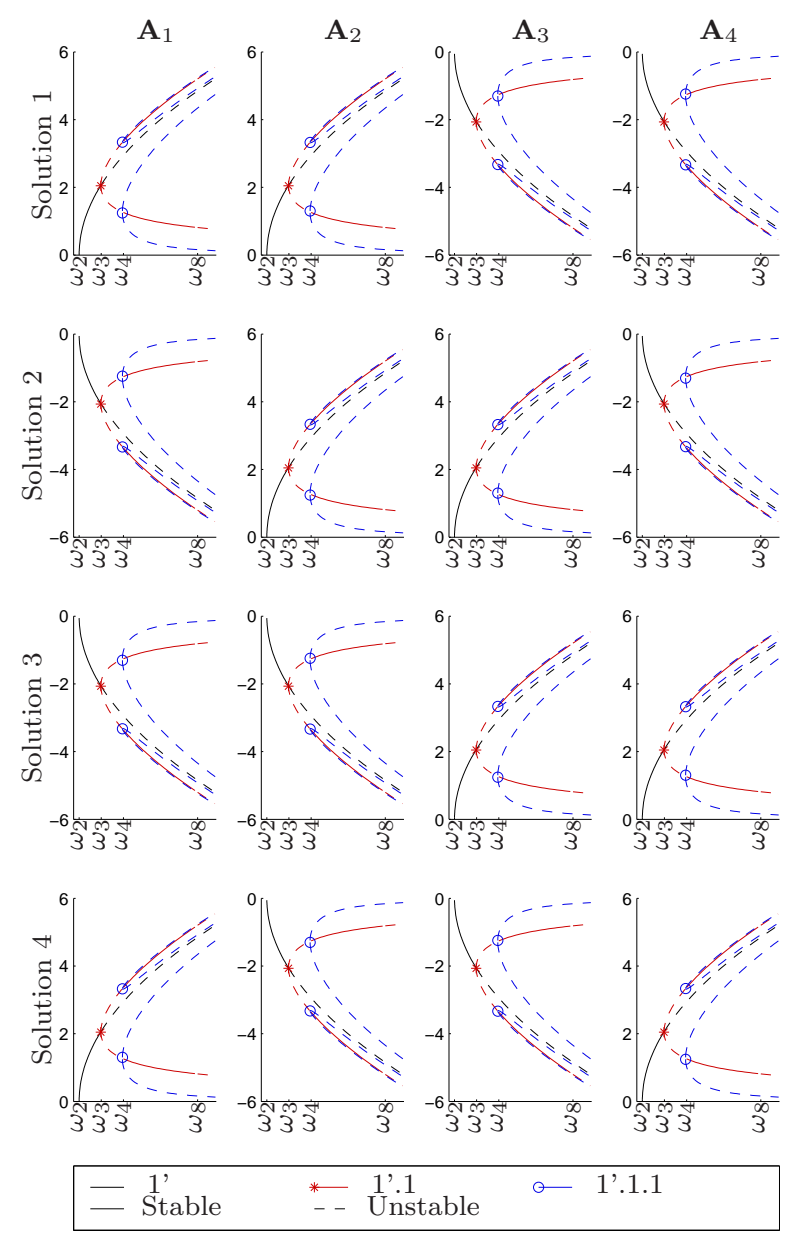

Figure 8: Solutions matching energy branch 1' and their bifurcations 


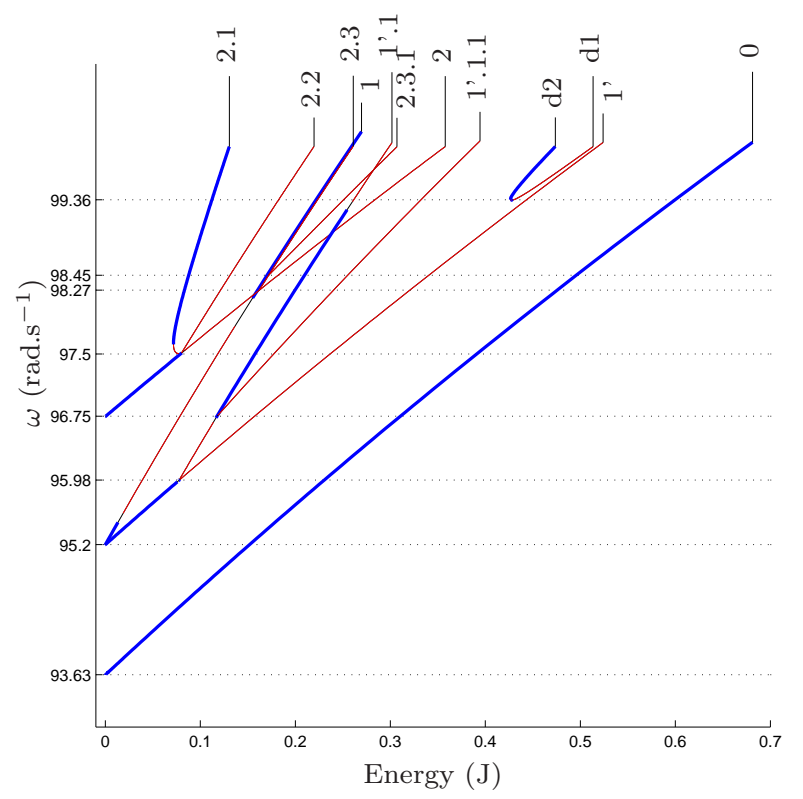

— Stable — Unstable

Figure 9: Backbones FEP with stability 


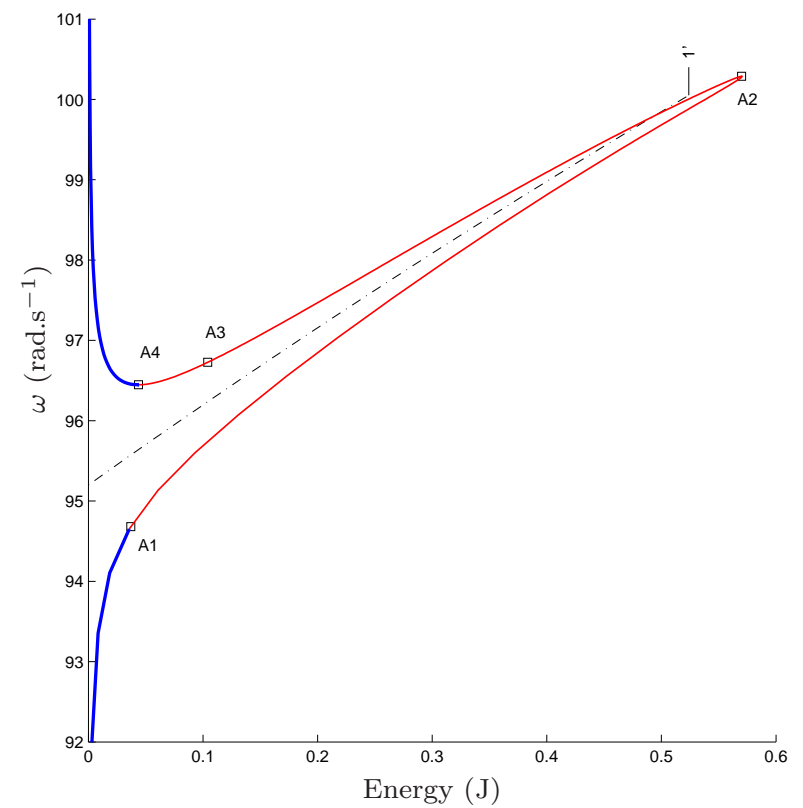

Figure 10: Forced case: Sol-A (positioned around branch 1') and its bifurcation points 


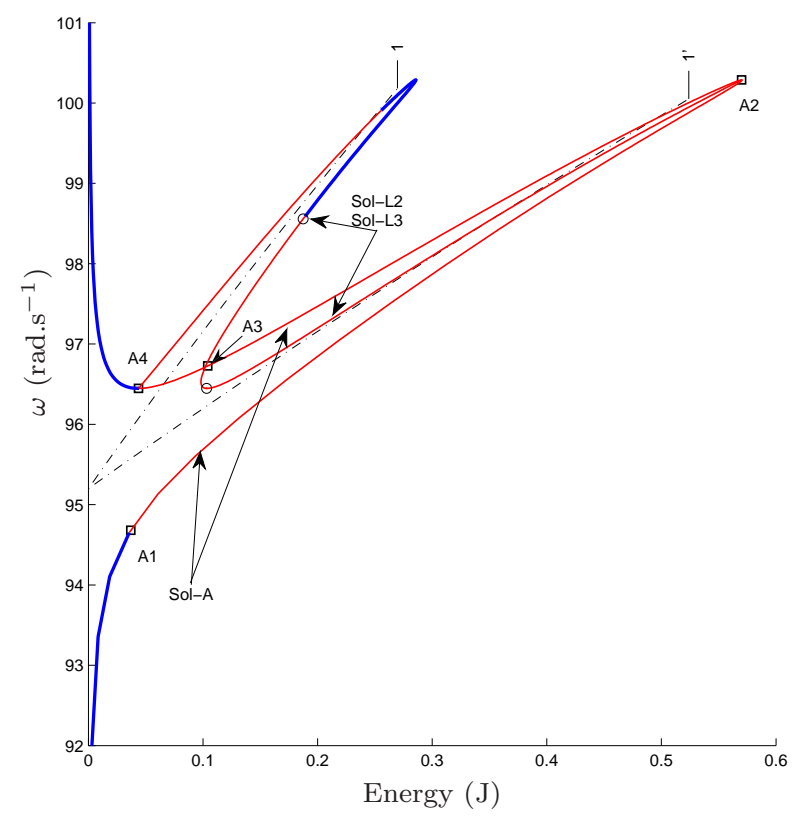

Figure 11: Forced case: Sol-A and its bifurcated branch SolL2 and Sol-L3 (positioned around branch 1) 


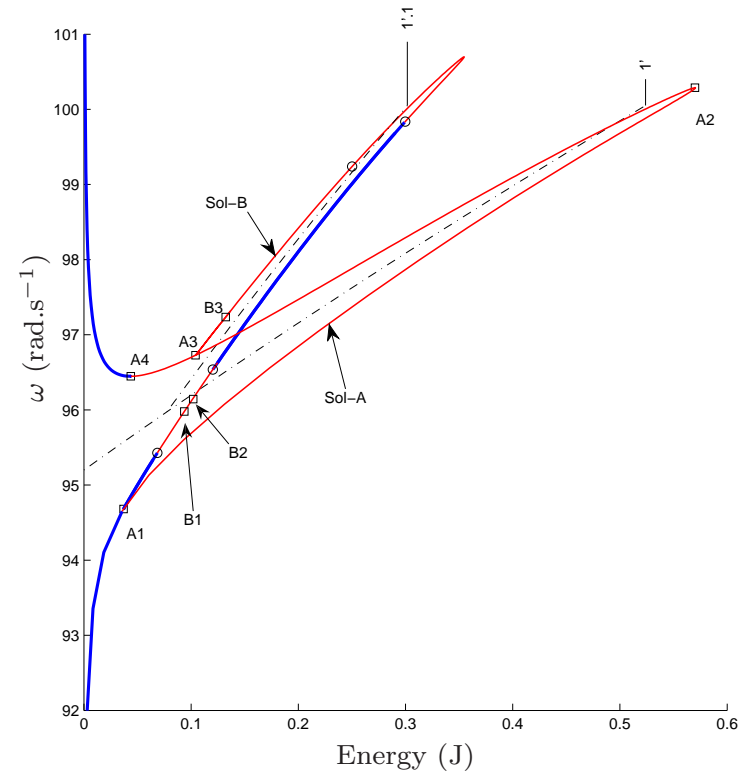

Figure 12: Forced case: Sol-A and its bifurcated branch Sol$\mathrm{B}$ (positioned around branch 1'.1) 


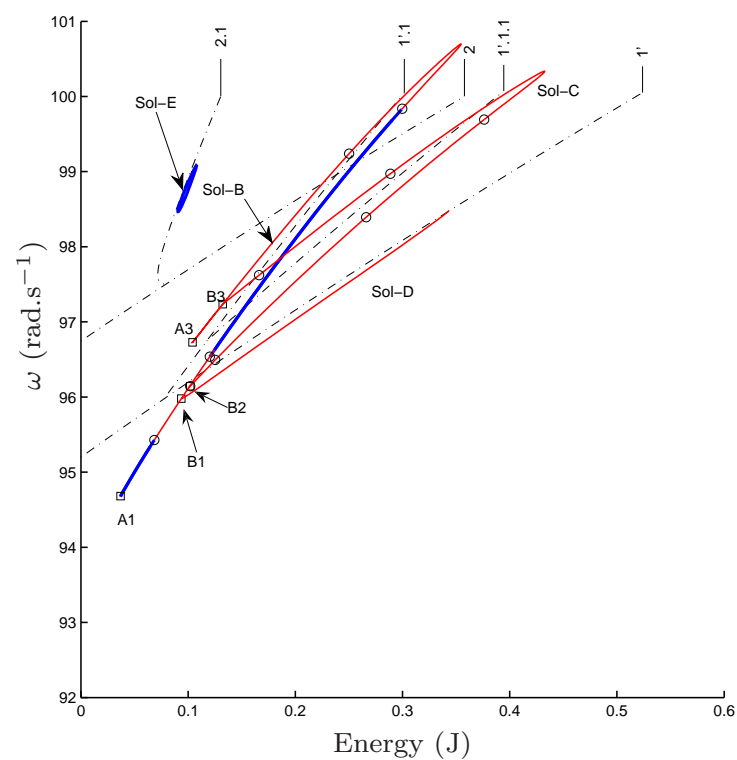

Figure 13: Forced case: Sol-B and its bifurcated branches Sol-C (positioned around branch 1'.1.1) and Sol-D (positioned near branch 1') in addition with the disconnected solution Sol-E (positioned near branch 2.1) 


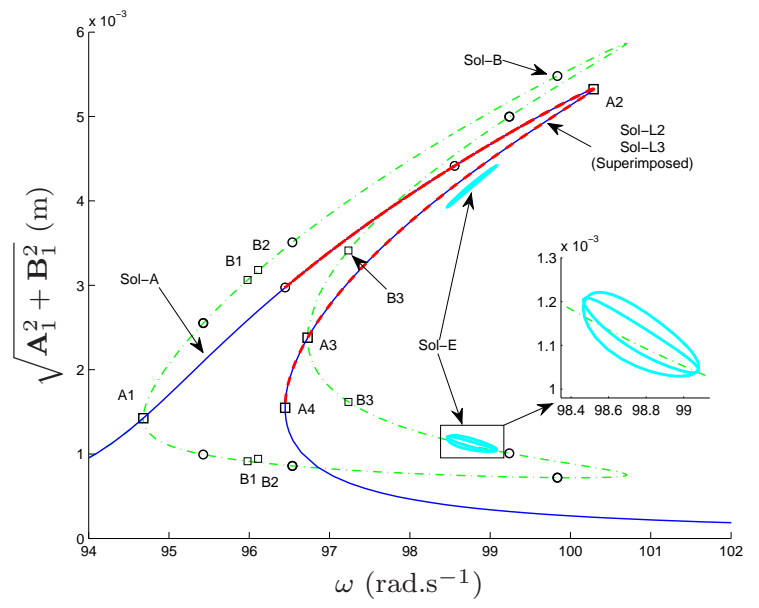

Figure 14: Solutions of the forced case in the frequencyamplitude plot for sector 1: (-) Sol-A, (- ) Sol-B, (- -) Sol-L2/Sol-L3, (-) Sol-E 


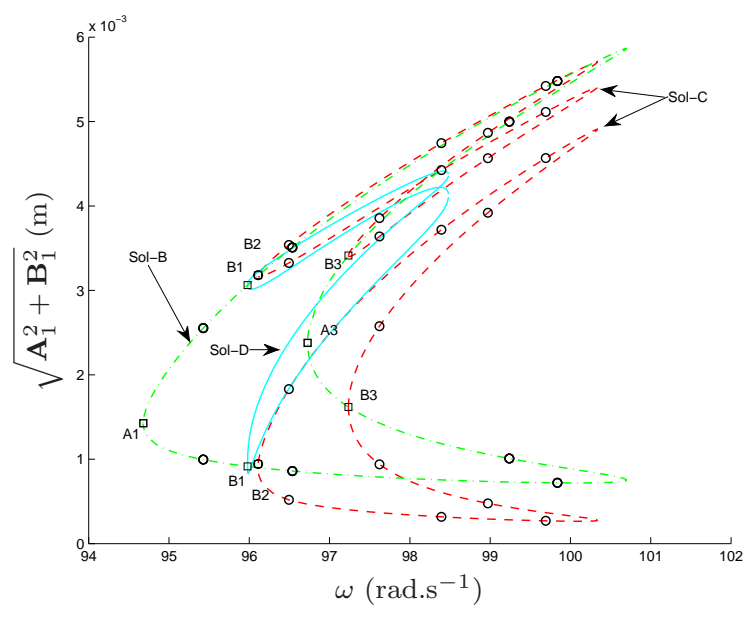

Figure 15: Solutions of the forced case in the frequencyamplitude plot for sector 1: (- .) Sol-B, (- -) Sol-C, (-) Sol-D 

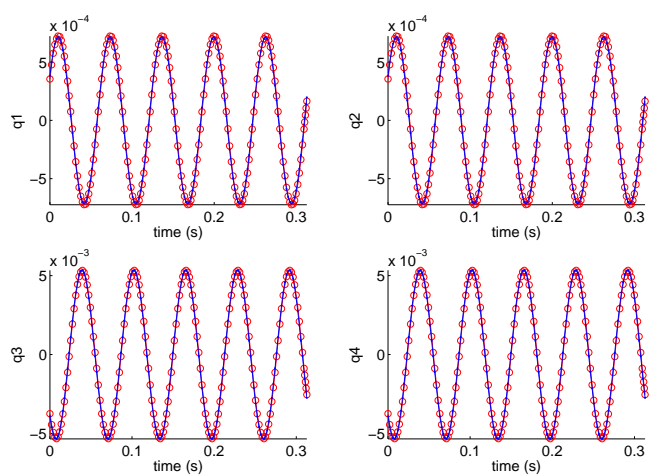

Figure 16: Comparison between HBM and numerical integration for a stable point of Sol-B at $\omega=99.53$ : (·) Numerical integration, (०) HBM solution 

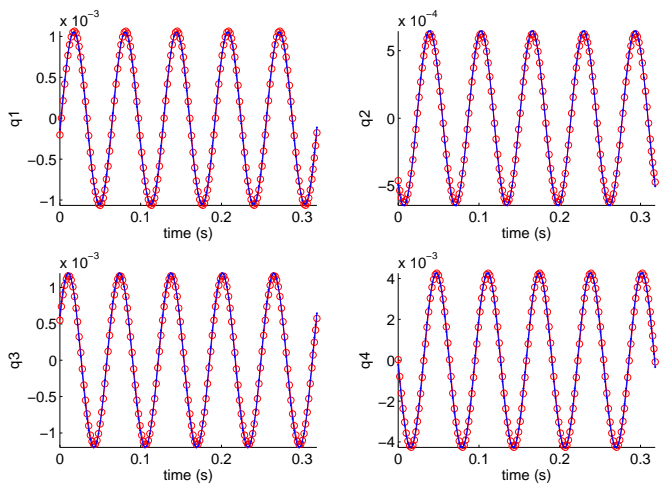

Figure 17: Comparison between HBM and numerical integration for a stable point of Sol-E at $\omega=98.84$ : (·) Numerical integration, (०) HBM solution 


\section{List of Tables}

1 Backbones branches characterization sum up 34 


\begin{tabular}{lccc} 
Branch & Nsol & $\omega_{i}\left(\right.$ rad.s $\left.^{-1}\right)$ & Sim/Non-sim \\
\hline 0 & 2 & $\omega_{1}=93.63$ & Similar \\
\hline 1 & 4 & $\omega_{2}=95.20$ & Similar \\
\hline $1^{\prime}$ & 4 & $\omega_{2}=95.20$ & Similar \\
\hline $1^{\prime} .1$ & 8 & $\omega_{3}=95.98$ & Non-similar \\
\hline $1^{\prime} .1 .1$ & 16 & $\omega_{4}=96.75$ & Non similar \\
\hline 2 & 2 & $\omega_{4}=96.75$ & Similar \\
\hline 2.1 & 8 & $\omega_{5}=97.50$ & Non similar \\
\hline 2.2 & 8 & $\omega_{5}=97.50$ & Non similar \\
\hline 2.3 & 4 & $\omega_{6}=98.27$ & Non similar \\
\hline 2.3 .1 & 8 & $\omega_{7}=98.45$ & Non similar \\
\hline $\mathrm{d} 1$ & 8 & $\omega_{8}=99.36$ & Non similar \\
\hline $\mathrm{d} 2$ & 8 & $\omega_{8}=99.36$ & Non similar \\
\hline
\end{tabular}

Table 1: Backbones branches characterization sum up 